\title{
Clostridium difficile infection in children - clinical, diagnostic and therapeutic aspects
}

\author{
Elena Cristina Popescu ${ }^{1 *}$, Rozina lagăru', Monica Luminos ${ }^{1,2}$, Dorina Duma', Adina Stăncescu', Anuța Bilaşco ${ }^{1}$, \\ Georgeta Constantinescu', Elena Gheorghe', Luminița Marin 1,2, Gheorghiță Jugulete ${ }^{1,2}$, Anca Drăgănescu' , \\ Angelica Vişan ${ }^{1,2}$, Camelia Kouris ${ }^{1}$
}

From The 9th Edition of the Scientific Days of the National Institute for Infectious Diseases Prof Dr Matei Bals Bucharest, Romania. 23-25 October 2013

\section{Background}

Clostridium difficile is the most common cause of antimicrobial-associated diarrhea and is a common healthcare associated pathogen. While asymptomatic carriage remains high among infants, recent epidemiological surveillance has demonstrated a rise in the prevalence of Clostridium difficile infection in children.

\section{Methods}

We present 4 cases of children admitted to the National Institute for Infectious Diseases "Prof. Dr. Matei Balş" in the last year with Clostridium difficile colitis; the children had ages between 2 and 12 years. Diagnosis of infection was based on the presence of three or more stools in a 24 hour period, isolation of the organism and detection of toxins $\mathrm{A} / \mathrm{B}$ in a diarrheal stool specimen (stool culture and PCR assay). We studied the clinical aspects and evolution after treatment.

\section{Results}

All children presented an important risk factor for Clostridium difficile infection: antimicrobial therapy prior to infection. Three children presented chronic pediatric comorbidities: one case of Ewing's sarcoma; one case of gastroesophageal reflux disease, recent abdominal surgery for gastric volvulus, use of proton pump inhibitors; one case of milk protein allergy. The clinical signs and symptoms were: fever (3 cases), watery diarrhea (2 cases), bloody diarrhea ( 2 cases), cramping abdominal pain, vomiting, loss of appetite and malaise.
The children received antimicrobial therapy: oral metronidazole in 2 cases, oral vancomycin in 2 cases, supportive treatment and probiotics, with good outcome. All patients recovered without complications. Relapse occurred in the case with osteosarcoma, after cytostatic treatment and he was treated with oral vancomycin. Rifaximin prophylaxis associated to the next cytostatic treatment prevented another relapse.

\section{Conclusion}

1. The diagnosis of Clostridium difficile colitis should be suspected in patients with diarrhea and associated risk factors, even in the pediatric population.

2. The increasing incidence of Clostridium difficile infection requires the implementation of appropriate policies for antibiotic use and further studies investigating optimal therapeutic and preventive strategies.

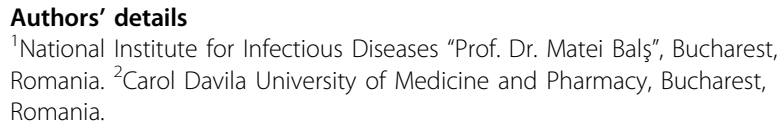

Published: 16 December 2013

doi:10.1186/1471-2334-13-S1-P46

Cite this article as: Popescu et al:: Clostridium difficile infection in

children - clinical, diagnostic and therapeutic aspects. BMC Infectious

Diseases 2013 13(Suppl 1):P46.

\footnotetext{
* Correspondence: drcristinapopescu@yahoo.com

${ }^{1}$ National Institute for Infectious Diseases "Prof. Dr. Matei Balş", Bucharest,

Romania

Full list of author information is available at the end of the article
} 